11

6

\title{
The Swiss Narcolepsy Scale (SNS) and its Short Form (sSNS) for the discrimination of narcolepsy in patients with hypersomnolence: $A$ cohort study based on the Bern Sleep-Wake Database
}

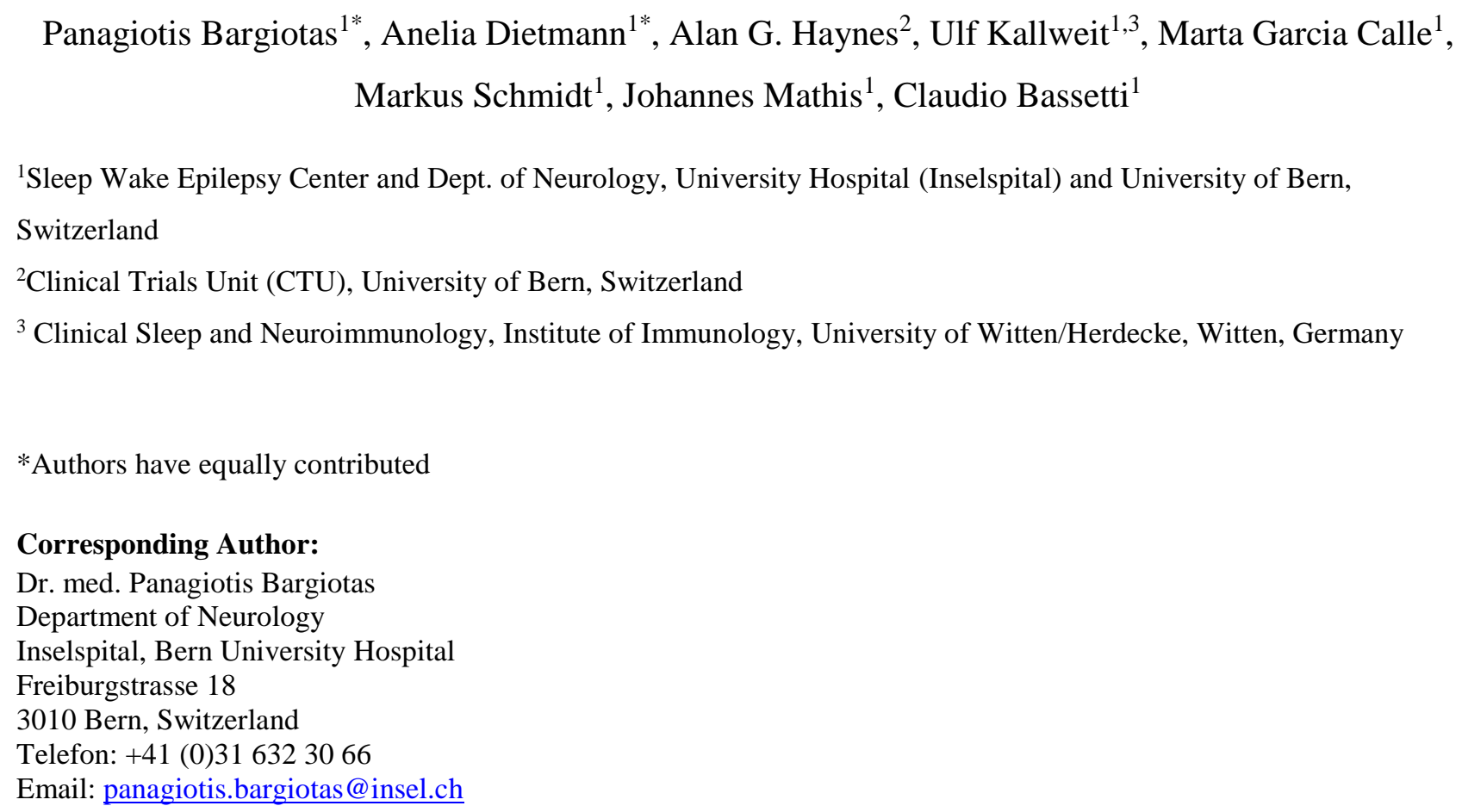

Acknowledgements: We thank Corinne Roth, Sandra Röthlisberger and Tanja Gerber for their assistance with the data transfer to the Bern Sleep-Wake Database. We also thank Christian Sturzenegger for the consultation.

Keywords: hypersomnolence, hypersomnia, screening tool, excessive daytime sleepiness, validation 


\section{ABSTRACT}

Previous studies reported high sensitivity and specificity of the Swiss Narcolepsy Scale (SNS) for the diagnosis of narcolepsy type 1 . We used data from the Bern Sleep-Wake Database to investigate the discriminating capacity of both the SNS and the Epworth Sleepiness Scale (ESS) to identify narcolepsy type 1 and type 2 in patients with central disorders of hypersomnolence (CDH) or sleepy patients with obstructive sleep apnea (OSA). In addition, we aimed to develop a simplified version of the SNS. We created the two-item short-form SNS (sSNS), based on the discriminative capability of the models including all possible combinations of the five questions of the SNS.

Using the previously published co-efficiencies, we confirmed the high capacity of the SNS in identifying narcolepsy type 1. The updated SNS (based on new co-efficiencies and cut-off) and the sSNS showed high capacity and were both superior to ESS in identifying narcolepsy type 1 . The sSNS correlated significantly with the SNS $(r=-0.897, p<0.001)$. No scale showed sufficient discrimination for narcolepsy type 2 .

This is the largest cohort study that confirms the discriminating power of SNS for narcolepsy type 1 in patients with hypersomnolence and the first study to assess its discriminative power for narcolepsy type 2. The easy-to-use and easy-to-calculate short-form scale has a high discriminating power for narcolepsy type 1 and may be used as screening tool, especially among general practitioners, to identify patients and accelerate their referral to a center of expertise. 


\section{INTRODUCTION}

Narcolepsy belongs to the group of central disorder of hypersomnolence (CDH) and is clinically characterized by excessive daytime sleepiness (EDS), cataplexy (in narcolepsy type I, NT1), hypnagogic or hypnopompic hallucinations, sleep paralysis and sleep fragmentation[1].

Narcolepsy has an estimated prevalence of $0.05 \%$ and symptom onset typically peaks during the second decade of life[2,3].

There is evidence for a delayed referral of patients with narcolepsy to a specialized center[4] and delayed diagnosis of narcolepsy[5,6] often due to lack of recognition of signs and symptoms and lack of knowledge about CDH in a broad medical community[4].

The correct diagnosis of narcolepsy is based on clinical features and objective measures including multiple sleep latency test (MSLT), polysomnography, and/or measurement of decreased or absent hypocretin in cerebrospinal fluid supported by HLA DQB1*0602 testing. Questionnaires such as the Ullanlinna Narcolepsy Scale (UNS)[7], Narcolepsy Severity Scale[8] and the Epworth Sleepiness Scale (ESS)[9,10] are frequently used in screening of EDS as well as evaluation of treatment effects in narcolepsy, but have a limited discriminative capability especially among patients with hypersomnolence[9-12]. ESS allows calculating a score that quantifies daytime sleepiness, respectively how likely participants fall asleep in different passive situations. It consists of 8 items with a 4-step rating scale. Total score ranges from 0 to 24. Excessive daytime sleepiness is defined by an ESS value $\geq 10[13,14]$.

In 2004, we introduced a new scale, the Swiss Narcolepsy Scale (SNS)[12] aiming to develop a simple, short and specific screening questionnaire for identifying patients with narcolepsy. The SNS is a selfadministered narcolepsy screening instrument which contains five questions and assesses the following parameters: (1) the inability to fall asleep; (2) feeling unrefreshed in the morning; (3) taking a nap at noon; (4) weak knees/buckling of the knees during emotions such as laughing, happiness, or anger and (5) sagging of the jaw during emotions such as laughing, happiness, or anger. A calculated value (with defined multipliers) of $<0$ is indicating the presence of narcolepsy[12].

This initial study compared the SNS with the UNS in 57 patients with NT1, 56 with non-narcoleptic hypersomnolence, and 40 healthy controls, and reported high sensitivity (96\%) and specificity (98\%) for NT1 compared to a similar sensitivity (98\%) but lower specificity (56\%) of the UNS[12]. This initial study and a recent validation study[15] focused mainly on the diagnostic accuracy of SNS in detecting NT1 against other types of hypersomnolence. Studies comparing the discriminating power of SNS and ESS for NT2 among patients with hypersomnolence are lacking.

In the current study, we aim 1) to assess the capacity of the SNS and the ESS in discriminating NT1 and NT2 in a larger cohort of new patients with disorders of hypersomnolence, 2) to provide an update 
version of the SNS based on new scoring coefficients and optimal cut-off point and 3) to develop a simplified short-form of the SNS to increase practicability in a daily practice of a broad community of physicians. In an effort to increase applicability of the SNS and mainly of the sSNS in a general practitioner's usual practice, we additionally assessed the discriminative power of the scales in an expanded cohort that included also patients suffering from obstructive sleep apnea, one of the most common causes of excessive sleepiness among patients who visit a general practitioner.

\section{METHODS AND PATIENTS}

This is a retrospective cohort study based on data from the Bern Sleep-Wake Database (Dietmann et al submitted). The protocol for the establishment of the database for clinical and research purposes was approved by the local ethics committee (Kantonale Ethikkommission Bern, 2016-00409). For this study, data sets collected between 2001 and 2016 for clinical purposes were used. Patients have been admitted to the Sleep-Wake-Epilepsy Center, Department of Neurology, Inselspital, University Hospital Bern, Bern, Switzerland for evaluation of suspected disorder of hypersomnolence. Patients underwent clinical routine work-up including clinical consultations and electrophysiological examinations (polysomnography, multiple sleep latency test, maintenance of wakefulness test, psychovigilance test and actigraphy), all patients filled in a set of questionnaires related to sleep-wakedisorders. Final diagnosis was reviewed for this study by two independent sleep specialists (A.D. and M.G.C.) according to the International Classification of Sleep Disorders (ICSD-3)[16] using medical history from hospital records, laboratory data, electrophysiological work-up of subjective complaints (including PSG, MSLT, MWT and actigraphy) and a battery of sleep-wake questionnaires. Patients included in this study were diagnosed with narcolepsy (type 1 and 2) or other disorder of hypersomnolence, including idiopathic hypersomnia, hypersomnolence due to a medical disorder, hypersomnolence associated with a psychiatric disorder, insufficient sleep syndrome, long sleepers, EDS of unknown origin and sleepy (ESS $>10)$ patients who have completed the SNS scale and were diagnosed with obstructive sleep apnea (Apnea-Hypopnea Index, AHI $>5 / \mathrm{h}$ ).

\section{Clinical Assessment}

The assessment of the clinical and epidemiological sleep-wake profile of the patients was based on the "Bern Sleep Questionnaire". The questionnaire contains 110 questions about demographics, reasons for referral to sleep laboratory, general information about sleep-wake habits and sleep problems, breathing and circulation, parasomnias and potential trigger factors, dreaming, waking-up, tiredness and sleepiness, cataplexy, hallucinations, stress, well-being, drugs, medication, neurological, psychiatric and other comorbidities, as well as information on family history. Furthermore, all questions included in the ESS, SNS and UNS are included in the Bern Sleep Questionnaire. 
1

22

3

4

54

75

8

96

10

117

12

138

14

$15^{9}$

1610

181

19

292

21

2213

23

244

265

2716

297

30

3118

32

$33^{19}$

34

350

361

37

382

4 (2)

41

424

43

425

456

4727

498

50

5129

53

540

55

561

57

582

59

61

$62^{34}$

\section{Statistical model}

To assess the diagnostic accuracy of the SNS, sensitivity and specificity were calculated for the cutoff value of 0 . Additionally, logistic regression was used to model the effect of the SNS on the probability of NT1 and NT2 separately. Model coefficients of the individual questions in the SNS were also re-assessed using logistic regression. The Hosmer-Lemeshow goodness-of-fit test and the Brier score were subsequently calculated to test for calibration and agreement between diagnosis and prediction, respectively. Complete cases analyses were performed.

To derive the new short-form SNS (sSNS), logistic regression and AUC for all combinations of questions was used to rank the discriminative ability of the combinations of questions. Although models with four and five questions typically showed higher discriminative abilities, a two question model (including at least one question on cataplexy and one question on awakening) was desirable. The best two-question model was chosen for further analyses. The cut-off for predicting narcolepsy was selected by summing the sensitivity and specificity for each possible predicted value from the model and selecting the cut-off with the largest sum. Cut-offs reported are on the linear predictor scale. Validation of the sSNS for NT1 and the re-parameterized SNS to predict NT1 was assessed via internal bootstrap validation to estimate the optimism in the AUC, Brier score and calibration plot slope and intercept. Briefly, a training sample was drawn and the model was refit in that sample, with Briers score and a test of calibration plot intercept and slope calculated for the training sample. The statistics from that training model were then compared to the original sample for an estimate of the test performance. Optimism was then calculated based on the difference between training and test performance. This procedure was performed 2000 times and the average optimism subtracted from the statistics from the original model to derive a corrected performance.

As an exploratory analysis, the best cut-off for ESS to determine NT1 and NT2 was assessed by calculating sensitivity and specificity at each possible cut-off (each value between the minimum and maximum), summing the sensitivity and specificity and determining the cut-off with the highest sum. AUC was also calculated.

Analyses were performed in Stata 15.1 and R 3.4.2 


\section{RESULTS}

\section{Patients}

In our data set, we identified 299 individuals with a disorder of hypersomnolence who have completed the SNS scale. The final cohort consisted of patients with NT1 (30\%), NT2 (7\%), idiopathic hypersomnia (15\%), hypersomnolence due to a medical condition or a substance (5\%), hypersomnolence associated with a psychiatric disorder (26\%) and hypersomnolence of unknown origin (17\%). Mean age was 33 years (range 23-48) and the male/female ratio was 1.45. For NT1 and NT2 mean age was 31 (range 23-42) and 25 years (range 19-34), and the male/female ratio was 2.0 and 2.2 respectively. Table 1 presents the demographic and clinical characteristics of the patients with $\mathrm{CDH}$.

Expanding the cohort of patients who completed the SNS and including not only patients with CDH but also sleepy $(\mathrm{ESS}>10)$ patients with obstructive sleep apnea $(\mathrm{AHI}>5 / \mathrm{h})$ we could identify 473 individuals.

\section{The discriminating power of the SNS for NT1 and NT2}

In our cohort, the SNS showed a sensitivity of $86 \%$ and a specificity of $88 \%$ in detecting NT1 against other types of hypersomnolence using the previously published scoring coefficients and the published cut-off of zero[12]. A Brier score of 0.87 indicated relatively poor agreement between observed and predicted outcome (NT1), although the Hosmer-Lemeshow test $(\mathrm{p}=0.70)$ was not statistically significant suggesting a normal calibration (Table 2). For the detection of NT2 against other types of hypersomnolence the SNS showed a sensitivity of $25 \%$ and a specificity of $71 \%$ (Table 2). The distribution of the SNS values in patients with NT1 are shown in Figure 1.

\section{The discriminating power of the updated SNS for NT1 and NT2}

Based on this new cohort, we recalculated the published model coefficients for scoring SNS and determined a new cut-off point of -1.83 . The parameterization of the model to predict NT1 (rather than not-NT1, as in the original parameterization) resulted in the following formula:

$$
Q 1 \times(-0.47)+Q 2 \times(-0.83)+Q 3 \times 0.58+Q 4 \times 0.56+Q 5 \times 1.45-2.75 \geq-1.83
$$

The sensitivity of the updated SNS for detecting NT1 against other types of hypersomnolence was $91 \%$ and the specificity $82 \%$. Brier score was 0.07 , indicating good agreement between observed and predicted outcomes (NT1). The Hosmer-Lemeshow test was also non-significant ( $p=0.39)$ suggesting a normal calibration (Table 2). 
The updated SNS showed a sensitivity of $63 \%$ and a specificity of $70 \%$ in detecting NT2 against other types of hypersomnolence, using the revised scoring coefficients and the revised cut-off of -2.75 (Table 2).

\section{The discriminating power of the simplified form SNS (SSNS) for NT1 and NT2}

The 2-item simplified form from the SNS (sSNS) was created based on the discriminative capability of the models including all possible combinations of the five SNS questions. Among them, the combination of SNS question 2 (feeling of being unrefreshed in the morning) and question 5 (reports of episodes with muscle weakness in the face/neck related to emotions) showed the highest discriminative capability for NT1 (supplementary table 1 and supplementary table 2). The accuracy for the sSNS in detecting NT1 was comparable to that of the SNS reaching $80 \%$ sensitivity and $92 \%$ specificity (Table 2). Brier score was 0.08, indicating good agreement, and the Hosmer-Lemeshow test was non-significant $(\mathrm{p}=0.26)$, suggesting a good calibration. The sSNS score correlate significantly with the updated SNS $(r=-0.89, \mathrm{p}<0.001)$.

Based on the sensitivity and specificity of the models to predict NT1 we determined a cut-off point of -0.68 for the sSNS. The parameterization of the model to predict NT1 resulted in the following formula:

$$
\mathrm{Q} 2 \times-0.82+\mathrm{Q} 5 \times 1.70-0.74 \geq-0.68
$$

The distribution of the sSNS values in patients with NT1 are shown in Figure 2. Model parameters are presented in Table 2.

We used the above formula to create an easy-to-use grid, based on the possible answers in sSNS (Q2 and Q5 of the SNS), in order to assess probability of NT1 diagnosis against other disorders of hypersomnolence (Figure 3).

None of the combinations among SNS questions showed sufficient capacity in discriminating NT2 within the cohort of patients with hypersomnolence (data not shown).

\section{The discriminating power of the original SNS, the updated SNS and the sSNS for NT1 among patients with CDH or OSA}

We then assessed the discriminating capability of SNS, updated SNS and sSNS to discriminate NT1 among patients with CDH or sleepy patients with obstructive sleep apnea (OSA). 
1 The original SNS, using the previously published scoring coefficients and the published cut-off of 12 zero[12], showed a sensitivity of $83.3 \%$ (95\% CI $73.1-90.2 \%)$ and a specificity of $90.6 \%$ (95\%CI

\section{The discriminating power of the ESS for NT1 and NT2}

In our cohort, the sensitivity of ESS score, using the typical cut-off of sleepiness (ESS $\geq 10$ ) was $68 \%$ and specificity was $56 \%$ for the identification of NT1 against other disorders of hypersomnolence (Table 3). By using various ESS cut-off's, finally applying the one (ESS $\geq 18$ ) with the best AUC, the sensitivity $(51 \%)$ and specificity $(78 \%)$ for identifying NT1 remained low.

Similarly, by using various ESS cut-off's, applying the one (ESS $\geq 10$ ) with the best AUC, the sensitivity for identifying NT2 against other disorders of hypersomnolence was $93 \%$ but specificity was very low (17\%). 


\section{DISCUSSION}

This is the largest known retrospective cohort study to assess the discriminative capacity of the SNS for narcolepsy. Our data confirmed the previously reported high capacity of the SNS in identifying NT1 against other types of hypersomnolence[12]. In addition, we updated the SNS by recalculating the model coefficients for scoring SNS and defining new cut-off scores for the scale. The capacity of the updated SNS for discriminating NT1 against other disorders of hypersomnolence was comparable to the capacity of the original SNS.

There is ample evidence for a delayed diagnosis of narcolepsy often due to the absence or lack of recognition of common narcolepsy symptoms, especially cataplexy. Therefore, the implementation of simple, easy-to-use, and reliable questionnaires on the symptom-based suspicion of narcolepsy and its subtypes in primary care may significantly increase the referral accuracy and improve resource utilization by narrowing the differential diagnosis upon referral. In an effort to increase its practicability, we aimed to simplify the SNS and introduced a simplified form (sSNS) which contains only two questions.

The sSNS correlated with the full SNS and demonstrated comparable validity with the SNS in detecting NT1 against other types of hypersomnolence. We created an easy-to-use grid, based on the sSNS formula, to simplify further the prediction of NT1, by selecting the relevant cell.

We then applied the original SNS, the updated SNS and the sSNS in a larger cohort of patients including sleepy patients with obstructive sleep apnea (OSA). The discriminative power of all scales, including the two-questions sSNS, remained satisfactory. This increases the applicability of the SNS and mainly of the sSNS in a general practitioner's usual practice, as OSA is one of the most common causes of pathological level of sleepiness among patients who visit a general practitioner.

Our data from the sSNS suggest that the combined reports of episodes with muscle weakness in the face/neck related to emotions and the subjective feeling of restorative night time sleep can better predict the NT1 among patients with hypersomnolence. Indeed, the cataplectic attacks and the restorative nature of sleep are considered, among sleep specialists, typical symptoms for NT (and specifically for NT1), in contrast to the absence of cataplexy in NT2 or to the non-restorative nature of sleep in other $\mathrm{CDH}$ such as idiopathic hypersomnia. However, often these two important sleepiness features are not implemented in the standard first-line screening of a sleepy patient.

Furthermore, we compared the capability of the SNS, the sSNS and the ESS to discriminate NT1 against other disorders of hypersomnolence. Our data further support the superiority of SNS and sSNS against ESS in identifying NT1, even if these higher cut-off ESS scores were used.

Finally, this is the first study to report the poor discriminative capacity of SNS for NT2. Both SNS and the updated SNS, showed low capacity in identifying NT2 against other disorders of hypersomnolence. No combination of the five SNS questions showed a satisfactory discriminative ability. Similarly, ESS 
showed low validity in identifying NT2 against other disorders of hypersomnolence even if the higher cut-off ESS scores were used. These findings are consistent with previous data showing that the distinction between narcolepsy without cataplexy (NT2) and other disorders of hypersomnolence (mainly the idiopathic hypersomnia) remains ambiguous, not seldom due to a diagnosis change over the time[17].

The retrospective approach and the small $\mathrm{N}$ number of patients with NT2 consist two important limitations of our study. Due of the retrospective design of the study, not all patients with CDH have completed the SNS, and therefore had to be excluded from the analysis. In addition, the small sample size of NT2 patients may have limited our ability to detect the discriminative power of the scales for NT2 in this cohort. Prospective cohort studies would overcome these limitations.

\section{CONCLUSIONS}

The SNS is a useful and valid complementary tool for the diagnosis of NT1 against other types of hypersomnolence. In this study, we introduce its short form (sSNS), a two-questions, simple, easy-touse, easier-to-calculate and reliable questionnaire in particular to be used in primary care as a screening tool for narcolepsy in patients with hypersomnolence. This could decrease the delay and increase the accuracy of referral of patients with hypersomnolence to a specialized sleep center for narcolepsy specific diagnostic. Finally, our data suggest that SNS and ESS are not the ideal tools for the discrimination of NT2 against other disorders of hypersomnolence.

Although further confirmatory studies and most importantly prospective studies on clinical biomarkers for disorders of hypersomnolence are needed, our data could be used for the improvement of diagnostic processes in these conditions and the development of more specific screening scales in the future. 


\section{Disclosures}

The authors thank Jazz Pharmaceuticals for funding of this study. Jazz Pharmaceuticals also reviewed the manuscript and had the opportunity to provide suggestions to the authors for their consideration. Although Jazz Pharmaceuticals reviewed the content of this manuscript, the ultimate interpretation, and the decision to submit it for publication was made by the authors independently.

"Jazz pharmaceuticals holds a royalty-bearing non-exclusive license to the SNS from the University of Bern"

Panagiotis Bargiotas has no specific conflict of interest with respect of present work. Dr. Bargiotas has received congress fees and travel reimbursements from Lundbeck Foundation.

Anelia Dietmann, Marta Garcia Calle, Markus Schmidt, and Johannes Mathis have no specific conflict of interest with respect of present work and have nothing to declare.

Alan G. Haynes is affiliated with CTU Bern, University of Bern, which has a staff policy of not accepting honoraria or consultancy fees. However, CTU Bern is involved in design, conduct, or analysis of clinical studies funded by not-for-profit and for-profit organizations. In particular, pharmaceutical and medical device companies provide direct funding to some of these studies. For an up-to-date list of CTU Bern's conflicts of interest see http://www.ctu.unibe.ch/research/declaration_of_interest/index_eng.html.".

Ulf Kallweit has received Honoraria for consultancy / advisory board and/or speaking engagements from: AOP Orphan Pharma; Bioprojet Pharma; Harmony Biosciences; Jazz Pharma; UCB Pharma

Claudio Bassetti has no specific conflict of interest with respect of present work. Prof. Bassetti has received honoraria for consultancy, lectures, and board memberships from the following institutions/companies: Jazz, Servier, UCB, Zambon, Cephalon Lundbeck, Pfizer Bohringer Ingelheim. His research is currently supported by grants of the following institutions/companies: Swiss National Science Foundation (SNF), ResMed, Respironics, Vifor Pharma, UCB Pharma, Schweizerische Herzstiftung, Tropos Stiftung, Parkinson Schweiz. 


\section{REFERENCES}

1. Scammell TE (2015) Narcolepsy. The New England journal of medicine 373 (27):2654-2662. doi:10.1056/NEJMra1500587

2. Dauvilliers Y, Montplaisir J, Molinari N, Carlander B, Ondze B, Besset A, Billiard M (2001) Age at onset of narcolepsy in two large populations of patients in France and Quebec. Neurology 57 (11):2029-2033

3. Ohayon MM, Ferini-Strambi L, Plazzi G, Smirne S, Castronovo V (2005) How age influences the expression of narcolepsy. Journal of psychosomatic research 59 (6):399-405. doi:10.1016/j.jpsychores.2005.06.065

4. Morrish E, King MA, Smith IE, Shneerson JM (2004) Factors associated with a delay in the diagnosis of narcolepsy. Sleep medicine 5 (1):37-41

5. Thorpy MJ (1992) The clinical use of the Multiple Sleep Latency Test. The Standards of Practice Committee of the American Sleep Disorders Association. Sleep 15 (3):268-276

6. Luca G, Haba-Rubio J, Dauvilliers Y, Lammers GJ, Overeem S, Donjacour CE, Mayer G, Javidi S, Iranzo A, Santamaria J, Peraita-Adrados R, Hor H, Kutalik Z, Plazzi G, Poli F, Pizza F, Arnulf I, Lecendreux M, Bassetti C, Mathis J, Heinzer R, Jennum P, Knudsen S, Geisler P, Wierzbicka A, Feketeova E, Pfister C, Khatami R, Baumann C, Tafti M, European Narcolepsy N (2013) Clinical, polysomnographic and genome-wide association analyses of narcolepsy with cataplexy: a European Narcolepsy Network study. Journal of sleep research 22 (5):482-495. doi:10.1111/jsr.12044

7. Hublin C, Kaprio J, Partinen M, Koskenvuo M, Heikkila K (1994) The Ullanlinna Narcolepsy Scale: validation of a measure of symptoms in the narcoleptic syndrome. Journal of sleep research 3 (1):5259

8. Dauvilliers Y, Beziat S, Pesenti C, Lopez R, Barateau L, Carlander B, Luca G, Tafti M, Morin CM, Billiard M, Jaussent I (2017) Measurement of narcolepsy symptoms: The Narcolepsy Severity Scale. Neurology 88 (14):1358-1365. doi:10.1212/WNL.0000000000003787

9. Hublin C, Kaprio J, Partinen M, Koskenvuo M, Heikkila K, Koskimies S, Guilleminault C (1994) The prevalence of narcolepsy: an epidemiological study of the Finnish Twin Cohort. Annals of neurology 35 (6):709-716. doi:10.1002/ana.410350612

10. Johns MW (2000) Sensitivity and specificity of the multiple sleep latency test (MSLT), the maintenance of wakefulness test and the epworth sleepiness scale: failure of the MSLT as a gold standard. Journal of sleep research 9 (1):5-11

11. Wing YK, Li RH, Ho CK, Fong SY, Chow LY, Leung T (2000) A validity study of Ullanlinna Narcolepsy Scale in Hong Kong Chinese. Journal of psychosomatic research 49 (5):355-361

12. Sturzenegger C, Bassetti CL (2004) The clinical spectrum of narcolepsy with cataplexy: a reappraisal. Journal of sleep research 13 (4):395-406. doi:10.1111/j.1365-2869.2004.00422.x

13. Bloch KE, Schoch OD, Zhang JN, Russi EW (1999) German version of the Epworth Sleepiness Scale. Respiration; international review of thoracic diseases 66 (5):440-447. doi:29408

14. Vignatelli L, Plazzi G, Barbato A, Ferini-Strambi L, Manni R, Pompei F, D'Alessandro R (2003) Italian version of the Epworth sleepiness scale: external validity. Neurological sciences : official journal of the Italian Neurological Society and of the Italian Society of Clinical Neurophysiology 23 (6):295-300. doi:10.1007/s100720300004

15. Christian Sturzenegger CRB, Gerd J Lammers, Ulf Kallweit, Wendy LM van der Zande, and Claudio L Bassetti (2018) Swiss Narcolepsy Scale : A simple screening tool for hypocretin-deficient narcolepsy with cataplexy. Clinical and Translational Neuroscience 2 (2)

16. AASM Manual for the Scoring of Sleep and Associated Events: Rules TaTS, ed 1. Westchester/IL, American Academy of Sleep Medicine,2007.

17. Trotti LM, Staab BA, Rye DB (2013) Test-retest reliability of the multiple sleep latency test in narcolepsy without cataplexy and idiopathic hypersomnia. Journal of clinical sleep medicine : JCSM : official publication of the American Academy of Sleep Medicine 9 (8):789-795. doi: $10.5664 /$ jcsm. 2922 
FIGURES CAPTIONS

1

2

3

4

6

Figure 1. Distribution of the SNS for NT1. Black points indicate correct predictions, grey points indicate incorrect (false negative) predictions. Sensitivity and specificity values are shown.

NT1, narcolepsy type 1; SNS, swiss narcolepsy scale

Figure 2 Distribution of the sSNS score for NT1 patients. Black points indicate correct predictions, grey points indicate incorrect (false negative) predictions. Sensitivity and specificity values are shown.

NT1, narcolepsy type 1; sSNS, short-form swiss narcolepsy scale

Figure 3 Grid to predict NT1. Black regions suggest the possibility of NT1. Values in the cell represent probability of NT1 against other types of hypersomnolence.

P: probability; NT1, narcolepsy type 1 


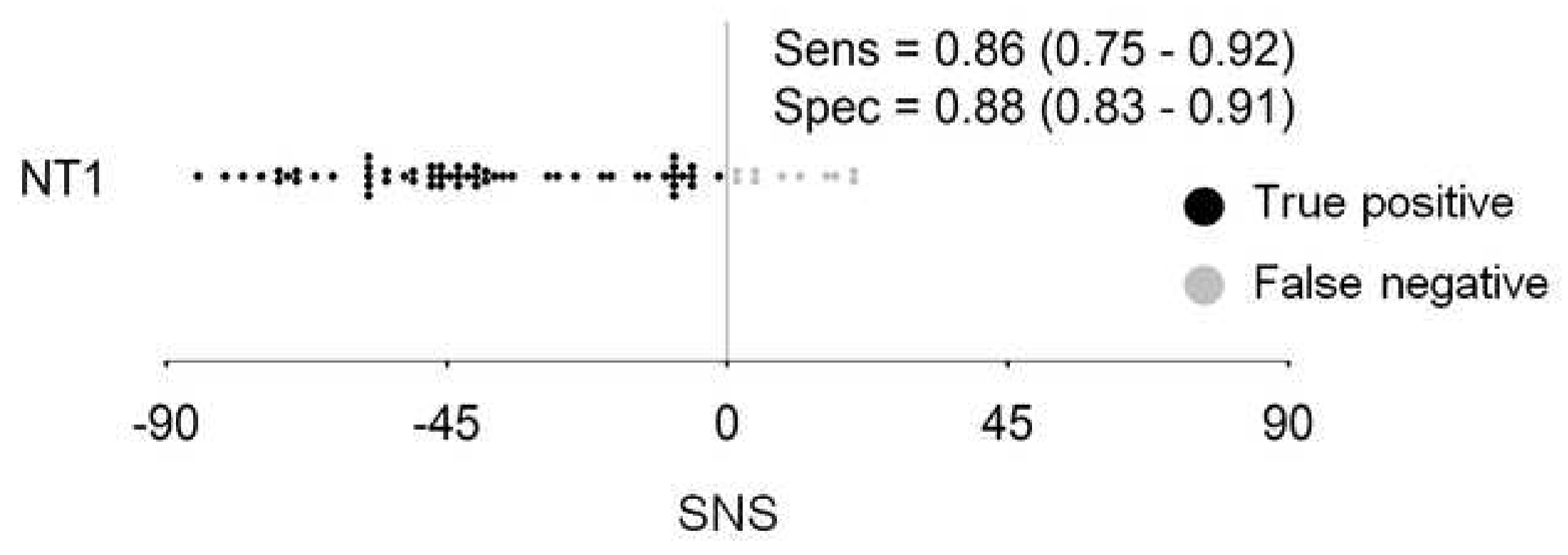




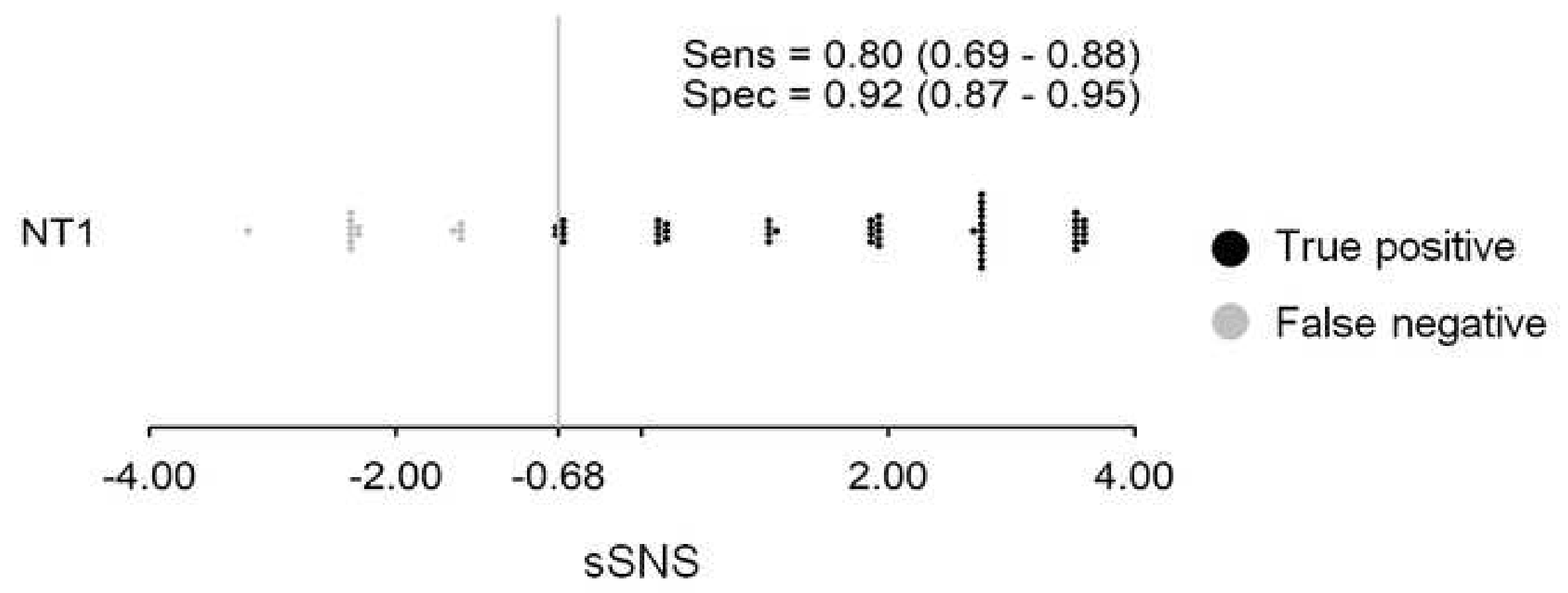


Q2: How often do you feel bad or not well rested in the morning?

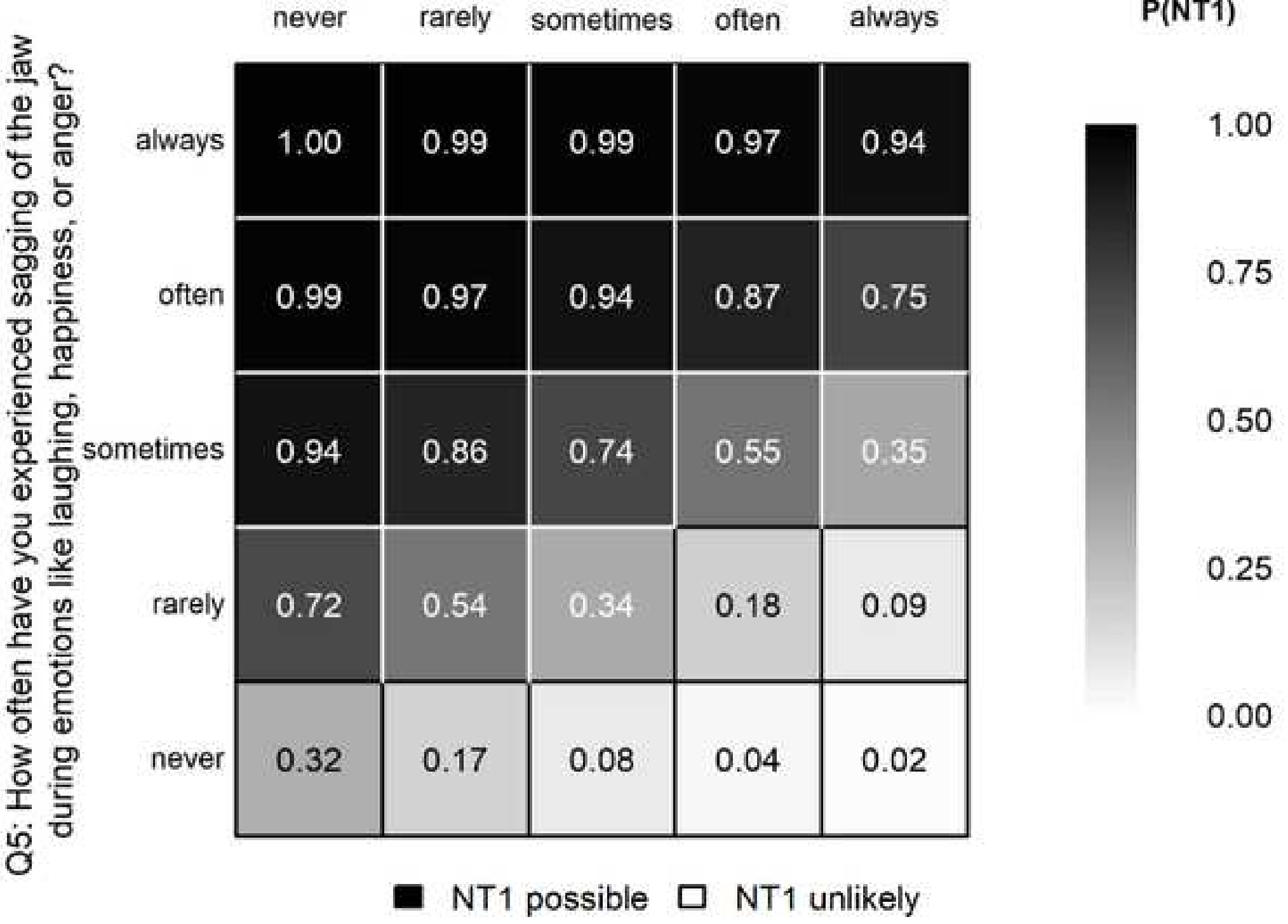




\begin{tabular}{|l|c|}
\hline \multicolumn{1}{|c|}{ Demographic and clinical characteristics } & n (\%) or median (lq, uq) \\
\hline Age & $32.9(22.8,47.7)$ \\
\hline Gender & $177(59 \%)$ \\
\hline male & $122(41 \%)$ \\
\hline female & $11.6( \pm 6.8)$ \\
\hline ESS mean score ( \pm SD) & $299(100 \%)$ \\
\hline & 69 \\
\hline Disorders of hypersomnolence & 16 \\
\hline NT1 & 35 \\
\hline NT2 & 12 \\
\hline Idiopathic hypersomnia & 2 \\
\hline Hypersomnia due to a medical disorder & 59 \\
\hline Hypersomnia due to a medication or substance & 91 \\
\hline Hypersomnia associated with a psychiatric disorder & 10 \\
\hline Insufficient sleep syndrome & 35 \\
\hline Long sleeper & \\
\hline EDS unclear etiology & \\
\hline $\begin{array}{l}\text { Table 1 Demographic and clinical characteristics of the cohort } \\
\text { LQ, lower quartile; UQ, upper quartiles; SNS, swiss narcolepsy scale; NT1, narcolepsy type } \\
\text { 1; NT2, narcolepsy type 2, EDS: excessive daytime sleepiness }\end{array}$ \\
\hline
\end{tabular}




\begin{tabular}{|c|c|c|c|c|c|c|}
\hline & \multicolumn{2}{|c|}{ SNS* } & \multicolumn{2}{|c|}{ Updated SNS*** } & \multicolumn{2}{|c|}{ SSNS } \\
\hline & NT1 & NT2 & NT1 & NT2 & NT1 & NT2 \\
\hline Cut-off & 0 & 0 & -1.83 & -2.75 & -0.68 & -2.26 \\
\hline Sensitivity (95\% CI ) & $\begin{array}{c}0.86 \\
(0.75-0.92) \\
\end{array}$ & $\begin{array}{c}0.25 \\
(0.10-0.49) \\
\end{array}$ & $\begin{array}{c}0.93 \\
(0.84-0.97) \\
\end{array}$ & $\begin{array}{c}0.63 \\
(0.39-0.82) \\
\end{array}$ & $\begin{array}{c}0.80 \\
(0.69-0.88) \\
\end{array}$ & $\begin{array}{c}0.44 \\
(0.23-0.67) \\
\end{array}$ \\
\hline Specificity (95\% CI ) & $\begin{array}{c}0.88 \\
(0.83-0.91) \\
\end{array}$ & $\begin{array}{c}0.71 \\
(0.65-0.76) \\
\end{array}$ & $\begin{array}{c}0.82 \\
(0.76-0.86) \\
\end{array}$ & $\begin{array}{c}0.70 \\
(0.64-0.75) \\
\end{array}$ & $\begin{array}{c}0.92 \\
(0.87-0.95) \\
\end{array}$ & $\begin{array}{c}0.83 \\
(0.78-0.87) \\
\end{array}$ \\
\hline $\begin{array}{l}\text { Hosmer-Lemeshow } \\
\text { goodness-of-fit }\end{array}$ & 0.70 & 0.02 & 0.39 & 0.28 & 0.26 & 0.00 \\
\hline Brier score & 0.87 & 0.87 & 0.07 & 0.05 & 0.08 & 0.22 \\
\hline AUC & 0.95 & 0.54 & 0.96 & 0.68 & 0.92 & 0.82 \\
\hline $\begin{array}{l}\text { Table } 2 \text { Sensitiviti } \\
\text { NT1 and NT2 in a } \\
\text { NT1, narcolepsy type I; } \\
\text { narcolepsy scale; sSNS, } \\
\text { *SNS in the current coho } \\
\text { **SNS in the current coh }\end{array}$ & $\begin{array}{l}\text { ind Specif } \\
\text { lort of ind } \\
\text { narcolepsy } \\
\text { t form SNS. } \\
\text { sed on the pr } \\
\text { ased on the u }\end{array}$ & $\begin{array}{l}\text { ties of SI } \\
\text { duals wit } \\
\text { II; CI, con }\end{array}$ & $\begin{array}{l}\text { nd its sh } \\
\text { persomn } \\
\text { ce interval; } \\
\text { coring coeff }\end{array}$ & $\begin{array}{l}\text {-form (s } \\
\text { ence } \\
\text { JC, area un } \\
\text { nts(Sturzen }\end{array}$ & $\begin{array}{l}\text { estimated } \\
\text { and Bassett }\end{array}$ & ;) \\
\hline
\end{tabular}




\begin{tabular}{|l|c|c|}
\hline \multirow{2}{*}{} & \multicolumn{2}{|c|}{ ESS } \\
\cline { 2 - 3 } & NT1 & NT2 \\
\hline Sensitivity (95\% CI ) & 0.56 & 0.96 \\
& $(0.46-0.65)$ & $0.82-0.99)$ \\
\hline Specificity (95\% CI ) & 0.68 & 0.07 \\
$(0.61-0.75)$ & $0.05-0.11)$ \\
\hline $\begin{array}{l}\text { Hosmer-Lemeshow } \\
\text { goodness-of-fit }\end{array}$ & 0.288 & 0.225 \\
\hline Brier score & 0.210 & 0.093 \\
\hline AUC & 0.681 & 0.501 \\
\hline
\end{tabular}

Table 3 Sensitivities and Specificities of ESS in discriminating NT1 and NT2 in the cohort of patients with hypersomnolence.

ESS Epworth Sleepiness Scale; NT1, narcolepsy type I; NT2, narcolepsy type II; CI, confidence interval; AUC, area under the curve 